Recherches \& Travaux

$64 \mid 2004$

Figures paradoxales de l'Auteur

\title{
Présentation. - Des paradoxes de la figure auctoriale
}

Chantal Massol

\section{OpenEdition}

12 Journals

Édition électronique

URL : http://journals.openedition.org/recherchestravaux/1187

DOI : 10.4000/recherchestravaux. 1187

ISSN : 1969-6434

Éditeur

UGA Éditions/Université Grenoble Alpes

Édition imprimée

Date de publication : 15 mai 2004

Pagination : 5-15

ISBN : 2-9518254-3-9

ISSN : 0151-1874

Référence électronique

Chantal Massol, «Présentation. - Des paradoxes de la figure auctoriale », Recherches \& Travaux [En ligne], 64 | 2004, mis en ligne le 20 mai 2019, consulté le 23 septembre 2020. URL : http://

journals.openedition.org/recherchestravaux/1187; DOI : https://doi.org/10.4000/recherchestravaux. 1187

(c) Recherches \& Travaux 
Chantal MASSOL

Université Stendhal-Grenoble 3

\section{Des paradoxes de la figure auctoriale}

L'auteur moderne (on entendra par là celui qui émerge au cours de la première moitié du XIX siècle, après la rupture historique de la Révolution) est nécessairement présent dans le champ de la représentation - mais, semble-til, pour la défier sans cesse. En témoigne la fréquence avec laquelle, du romantisme à l'époque la plus contemporaine, dans la littérature comme dans les arts, se présentent, et pas seulement comme des "faire-valoir", des images qui semblent aller à l'encontre de la création d'une figure d'autorité paradoxales, donc, au sens premier du terme: elles heurtent l'opinion commune.

Images qui surgissent tantôt à l'occasion des stratégies que déploient les auteurs réels, comme ces imagos paradoxales offertes à l'écrivain en quête d'identité par tel ou tel scénario auctorial (ceux du romantisme ironique, ou du romantisme désenchanté, pour le premier XIX' siècle), tantôt dans les textes eux-mêmes, où l'auteur se donne des hypostases parfois étonnantes en divers types de personnages qui le représentent par ce qui a priori ne saurait le définir : détournement de l'origine (éditeur, plagiaire, nègre...), défaut de garantie (faussaire, mystificateur...) ou de créativité ${ }^{I}$ (copiste, scribe, interprète, traducteur, voire ordinateur...) - en certains se rassemblent toutes ces négations. Figures, aussi, que profilent des conduites d'auteurs (l'auteur malgré lui, l'auteur mineur, l'auteur inconnu), ou qui s'inscrivent dans les textes, comme autant de constructions auxquelles participe le lecteur ${ }^{2}$, de feintes ${ }^{3}$.

I. L'auctor est celui qui garantit son propos; avant cela il est (sens premier de augeo) celui qui "produi $[\mathrm{t}]$ de l'existence" (É. Benveniste, Le Vocabulaire des fonctions indo-européennes, Minuit, 1969, II, vol. 2, ch. 6, p. I5I).

2. L'auteur induit.

3. La figure est ce que l'on forge, imagine. Le Dictionnaire bistorique de la langue française 
Ou, encore, fictions, qu'élaborent par exemple des dispositifs préfaciels (l'éditeur), que proposent d'eux-mêmes des écrivains (invention autobiographique), que des récits offrent d'auteurs connus ("fictions d'auteurs ${ }^{4}$ )...

Paradoxales, ces images, figures, ou fictions le sont parfois parce qu'en elles se joignent, de manière oxymorique, deux éléments contradictoires (le prophète-saltimbanque, l'auteur-enfant); parce qu'elles n'assurent pas l'effet pour lequel elles paraissent avoir été convoquées (l'autorité d'emprunt); le plus souvent, parce qu'elles paraissent signifier, à des titres et des degrés divers, un déficit de l'auctorialité, voire un déni de celle-ci.

On ne saurait, bien entendu, songer à faire du paradoxe un mode de manifestation spécifiquement moderne de la figure auctoriale. Il est bien clair, au contraire, que dès l'apparition - au Moyen Âge - de la "fonctionauteur", naissent des représentations qui participent d'un double jeu de consécration et de dérobade's, que, dans le temps même où cette figure entreprend de se construire, se mettent en place des dispositifs souvent ludiques qui œuvrent à sa déconstruction ${ }^{6}$. Elle se trouve ainsi, dès l'origine, investie d'une "fonction critique ${ }^{7}$ ", que les textes, au cours de l'histoire littéraire, font jouer de différentes manières, selon les types d'ambigütés ou de contradictions qui se rencontrent dans le processus de construction de l'auctorialité, mais qui lui est, on est tenté de le penser, essentielle.

C'est pour cela, sans doute, que l'époque moderne voit (c'est une hypothèse) le déploiement de ce "paradigme paradoxal " [A. Brunn, infra] : il est en rapport avec l'importance désormais accordée à la figure auctoriale, qui s'est affermie, à la fonction-auteur, qui s'est renforcée ${ }^{8}$. Point n'est besoin de

rappelle la parenté sémantique de figura et de fingere, "modeler dans l'argile", d'où provient en français "feindre». La figure de l'auteur se trouve ainsi définie, par M. Couturier, comme "l'auteur reconstruit comme principal sujet énonciatif du texte dans l'acte même de lecture", La Figure de l'auteur, Seuil, coll. « Poétique», 1995, p. 14.

4. La notion est définie, infra, par S. Rabau (voir, également, S. Rabau et S. Dubel, Fiction d'auteur? Le discours biographique sur l'auteur de l'Antiquité à nos jours, Champion, 2001).

5. Voir sur ce point l'ouvrage dirigé par N. Jacques-Lefevre avec la collaboration de F. Regard, Une histoire de la a fonction-auteur "est-elle possible?, actes du colloque organisé par le LiDiSa, E.N.S Fontenay-Saint-Cloud, mai 2000 , Publications de l'université de SaintÉtienne, 2001, et plus précisément l'article de F. Pomel, "La Fonction-auteur dans le Roman de la Rose de Jean de Meun: le double jeu de la consécration et de l'esquiven, p. 89-Io6.

6. J.-L. Diaz rappelle ici même le caractère transséculaire de la figure de l' "'auteur-malgrélui .....

7. N. Jacques-Lefevre, op. cit., "Présentation \#, p. 9.

8. Quoi de plus paradoxal d'ailleurs que la modernité (esthétique) elle-même? A. Compagnon a mis en évidence le paradoxe par lequel elle se définit, depuis le début du XIX" siecle (une "tradition de la rupture"), et ceux qui jalonnent son histoire (Les Cinq Paradoxes de la modernité, Seuil, 1990). 
rappeler longuement le tournant majeur que prend l'histoire de celle-ci à la fin du XVIII siècle et au début du XIX' $:$ R. Chartier, notamment, nuançant les affirmations de $\mathrm{M}$. Foucault ${ }^{9}$, a retracé les étapes de son invention, mis en évidence les dispositifs juridiques, répressifs, matériels ${ }^{\text {IO }}$ qui lui ont donné existence; à l'orée de la période qui est notre champ d'étude, elle est entièrement constituée, nécessaire, centrale ${ }^{\text {II }}$. Objet de savantes mises en œuvre, le paradoxe est alors si présent qu'il semble dominant - du moins en ce qui concerne la figure textuelle de l'auteur. Et ce, hors des contextes (romantisme ironique, symbolisme, surréalisme, "mort de l'auteur "...) où ce traitement paradoxal est attendu. On voit que cette figure entre volontiers en tension avec les discours auctoriaux les plus légitimants - même lorsqu'elle paraît en être une pure émanation: la figure du médecin, par exemple, semble des mieux accréditées dans la littérature réaliste-naturaliste; de fait, elle ne fonde pas la légitimité auctoriale (Balzac) - ou les imaginaires auctoriaux les plus valorisants: alors que les scénarios auctoriaux du romantisme, en plein moment de "sacre de l'écrivain", magnifient l'auteur, au point de l'élever au rang de monarque (Hugo "roi littéraire ${ }^{\mathrm{I} 2}$ ", Balzac "Napoléon des lettres»...), les textes tracent souvent des figures moins glorieuses, plus douteuses $^{13}$...

Les raisons d'être du paradoxe sont souvent conjoncturelles: il s'agit, en divers moments de rupture, ou d'évolution, de lutter contre une doxa auctoriale à dépasser (celle du classicisme, celle de la "mort de l'auteur")... Mais il est, plus fondamentalement, une "logique du paradoxe" [J.-L. Diaz, infra] qui s'exerce, sinon continûment, du moins avec une récurrence remarquable: c'est selon un paradoxe pragmatique que s'instaure, dans bien des cas, l'auctorialité; déclarer "je ne suis pas auteur" est alors l'acte même qui institue l'auteur, le dire venant contredire le dit ${ }^{\mathrm{I}}$. Comme l'œuvre elle-même,

9. "Qu'est-ce qu'un auteur? " (1969), Dits et écrits, I (1954-1969), éd. établie sous la direction de D. Defert et F. Ewald, Gallimard, 1994.

Io. R. Chartier, Culture écrite et sociêté. L'ordre des livres (XIV-XVII' siècles), ch. 2: "Figures de l'auteur", Albin Michel, 1996.

II. "La nouvelle économie de l'écriture suppose la pleine visibilité de l'auteur, créateur original d'une cuvre dont il peut légitimement attendre un profit" (R. Chartier, ibid., p. 57).

12. Voir à ce sujet J.-L. Diaz, "Quand le maître devient chef d'école", Romantisme, $\mathrm{n}^{\circ}$ I22, $4^{e}$ trimestre 2003 .

13. Figures inquiétantes, peu dignes de crédit, de l'ogre (Han d'slande), du diable (Le Rhin...), chez le premier (voir C. Raulet, "Hlugo ogre de son lecteur», groupugo.div.jussieu.fr); figures dévaluées du plagiaire, du rhapsode, du faussaire chez le second (Un prince de la Bohème, La Grande Breteche...).

I4. " [...] l'enveloppe énonciative vient contredire le contenu qu'elle est en train de délivrer par le fait même de le délivrer comme elle le fait" (D. Maingueneau, Pragmatique pour le 
qui s'engendre de la destruction qu'elle donne à voir, l'auteur semble se promouvoir selon le "paradoxe du phénix ${ }^{15}$ "...

A cette logique, des raisons structurelles, qui tiennent pour une bonne part au caractère institutionnel de la pratique de la littérature, aux particularités du champ littéraire. L'écrivain est "quelqu'un dont l'énonciation se constitue à travers l'impossibilité même de s'assigner une véritable "place" ${ }^{16}$ ". Pour exister, la littérature travaille, en effet, à se créer un lieu (les institutions qui permettent son exercice) tout en étant un effort pour se délocaliser (demeurer une véritable activité créatrice). La stratégie d'insertion de l'écrivain dans le champ littéraire passe, dès lors, par la quête d'une "localité paradoxale», résultant d'une négociation entre le lieu et le non-lieu d'une "paratopie», selon le terme forgé par D. Maingueneau ${ }^{17}$. D'où l'investissement des espaces qui, dans une société donnée, apparaissent comme frontaliers; d'où ces représentations de l'auteur qui prennent pour modèles des personnages qui semblent ne pas occuper de lieu fixe, ou être, à des titres divers, en marge: le saltimbanque, par exemple (les petits romantiques), l'errant (Nerval), le paria, l'exilé (Pinget), le clandestin, le pirate, le criminel, l'infâme (Schwob), l'enfant (la bande dessinée)... Cette fonction paratopique peut être assumée, comme on le verra, par des personnages en marge de l'auctorialité elle-même: copistes, scribes (Nerval, Schwob, Pinget, Calvino, Goscinny), "écrivassiers", "écrivailleurs" (Pinget)... Ces figures sont mises en jeu - elles ne composent pas un portrait statique. Le travail de la fiction consiste à les mettre au service de la quête auctoriale par la distanciation, le dépassement, le retournement du négatif en positif - par l'exploration et l'exploitation des tensions constitutives de toute paratopie (celle-ci étant par définition ambiguë, bipolaire, associant un élément minimal à un élément maximal (ex: le saltimbanque au prophète, l'obscur - Schwob - au «roi, philosophe et dieu»). Elles sont le moyen d'un passage à la limite, lieu d'où l'auctorialité peut tenter de se saisir et de se représenter (fût-ce par le biais d'une appréhension a contrario, ou au prix - Borges - d'une démonstration par l'absurde) et d'où peut se réfléchir le processus de son instauration.

discours littéraire, Bordas, 1990, p. 168). De manière semblable, en déclarant «je suis un traître ", * on montre qu'on est un homme de bien à travers l'énonciation même qui affìrme la culpabilité * (ibid., p. I64).

I5. D. Maingueneau, ibid., p. 168, et Le Contexte de l'œuvre littéraire. Énonciation, écrivain, societé, Dunod, I993, p. I7I.

16. D. Maingueneau, Le Contexte de l'auvre litteraire, ibid., p. 27.

17. Ibid., p. 28. 
C'est là, d'ailleurs, une démarche générale: nos figures sont, dans leur quasi-totalité, soumises à ce principe de renversement. L'on voit ainsi que le faussaire peut être un créateur authentique (Calvino), le plagiaire, un réinventeur (Borges); la figure de l'éditeur manifeste une volonté de maîtrise auctoriale sur le texte - un même désir de maîtrise se fait jour à travers les dérobades de l'auteur, au cinéma (Lynch); l'auteur inconnu rétablit, en lui donnant pour garantie l'intuition, la légitimité littéraire (Nodier); l'auteur sans œuvre vient à l'existence en se niant dans l'abolition de celle-ci (VilaMatas)...

De même, le passage à la limite est ce qui confere, dans l'ensemble, à la figure auctoriale, sa fonction critique ${ }^{18}$. Cette dernière s'exerce méthodiquement à l'égard de tous les aspects de la fonction-auteur, et révèle d'autres contradictions, qui sont inhérentes à celle-ci. Elle examine, d'abord, la nécessaire assignation d'origine du discours littéraire. A. Brunn a noté le fort "interdit d'auctorialité ${ }^{19}$ ", d'origine théologique, qui frappe l'auteur dans le temps même où sa figure émerge - un temps long, qui va jusqu'au début du XVIII ${ }^{c}$ siècle: l'Auteur par excellence, d'après la définition du Dictionnaire universel de Furetière, c'est Dieu ${ }^{20}$. Là réside l'un des paradoxes fondamentaux: l'énonciation divine, première, est un modèle (elle est le Modèle absolu); ce modèle, cependant, ne souffre pas l'imitation ${ }^{21}$. De cette contradiction essentielle, la figure de l'auteur porte trace encore ${ }^{22}$ à l'époque où elle est mieux fixée (de même qu'en porte trace le débat sur l'auteur: en plein $\mathrm{XX}^{\prime}$ siècle, Sartre et Mauriac s'affrontent sur ce point, le premier reprochant au second de tuer la liberté de ses personnages en adoptant une posture divine, et tentant d'esquisser dans ses propres romans la figure d'un auteur non-divin ${ }^{23}$ - ce qui laisse entier le paradoxe!). De la permanence de cet interdit initial, au-delà des époques théologiques, témoigne la figure (aussi

18. "Les paradoxes sont des monstres de la vérité", affirmait B. Gracián (1647), Art et figures de l'esprit, Seuil, 1983, p. 180. On sait le rôle du paradoxe dans la littérature moderne: il est l'expression du non-conformisme, du mépris des normes qu'affiche volontiers l'écrivain depuis le XIXe siècle. Et même dans la pensée moderne, y compris scientifique: renverser la doxa, geste provocateur, conduit, comme le souligne J.-J. Robrieux (Eléments de thétorique et d'argumentation, Dunod, 1993, p. 175) à des vérités.

19. L'Auteur, GF-Flammarion, "Corpus", 200I, p. 21.

20. "Qui a créé ou produit quelque chose. On le dit par excellence de la première cause qui est Dieu $[\ldots]$ ".

2I. Voir A. Brunn, op. cit., p. 22.

22. La définition du terme d' "auteur" par Littré n'est guère différente de celle de Furetière: "Cause première d'une chose. L'auteur de toutes choses, Dieu ".

23. Celle-ci trouve une de ses incarnations dans le personnage de Roquentin, dans La Nausée. 
ancienne que l'auctorialitć) du copiste - le texte originel à "copier" fût-il devenu pure absence; celle-ci dit assez que l'écrivain ne dispose pas d'emblée d'une voix auctoriale; il lui revient de créer les conditions de son émergence, depuis les marges de l'auctorialité sur lesquelles il est placé, par un travail du texte auquel cette figure ancillaire même se trouve soumise (Nerval). C'est encore la question du rapport à une écriture première que pose cette même figure, quand elle a à charge d'incarner l'idée d'une littérature qui serait essentiellement intertextualité, quand elle fait apparaître, comme l'a soutenu $\mathrm{R}$. Barthes, que «l'écrivain ne peut qu'imiter un geste antérieur, jamais originel ${ }^{24}$ n: cette butée ultime qu'est l'Énonciation première a totalement disparu à présent. Le paradoxe fondamental n'en disparaît pas pour autant, il ne fait que varier dans ses termes: la présence d'un Auteur originel frappait l'auctorialité d'une forme d'interdit; son absence la rend impossible, voire impensable... Il appartient alors à l'auteur-copiste de lutter victorieusement (Calvino, mais déjà, aussi, Nerval) contre sa propre "mort"... Ainsi qu’à l'auteur-plagiaire (Schwob, Calvino, Borges), faussaire (Calvino, Pinget), pasticheur (Calvino, Pinget), traducteur (Calvino), nègre (Pinget, Calvino), etc. Car les nombreuses figures qui évoquent la pratique d'une écriture seconde, et qui pour beaucoup relèvent du paradigme du faux, particulièrement bien représenté dans notre corpus - est-ce un effet de nos textes programmatiques? -, indiquent, comme celle du copiste, la difficile conquête, ou la problématique saisie d'une voix-origine.

Assigner une origine au discours, c'est faire de ce discours un objet d'appropriation - tel est pour M. Foucault ${ }^{25}$ le premier élément de définition de la fonction-auteur. Le rapporter, donc, à un sujet responsable, qui soit également un sujet justiciable. De là un autre paradoxe fondamental, clairement mis en évidence par M. Couturier ${ }^{26}$ : face à l'obligation de s'exposer, l'auteur est tenté de se détacher de son texte, de développer des statégies d'esquive. Depuis la fin du XVII" siècle, qui voit se préciser son statut juridique, il semble inextricablement pris dans une contradiction initiale - aux allures de double bind - provoquée tout à la fois par la nécessité de se mettre en avant (il en retire des avantages ${ }^{27}$ ) et le désir de se dérober. D'où cette

24. Ainsi le copiste (sous les traits, en l'occurrence, de Bouvard et Pécuchet) désigne "précisément", pour Barthes (i.e. paradoxalement), "la vérité de l'écriture " "La mort de l'auteur " (1968), Eutres complètes, nouvelle édition revue, corrigée et présentée par É. Marty, t. III, Seuil, 2002, p. 43 [cité par A.-M. Monluçon, infra]).

25. Art. cit.

26. Op. cit.

27. Notoriété, reconnaissance du talent, droits d'auteur... 
figure d' «auteur-en-fuite" que la fiction moderne ne cesse de profiler (on la voit se manifester ici dans le cinéma de Lynch). Elle entend déjouer la censure, et offrir un retranchement au sujet écrivant, qui se veut inaccessible. D'où ces portraits in figura (mais aussi ces conduites d'écrivains réels, exemplairement représentées ici par les "camouflages» et les dénégations de Nodier ${ }^{28}$ ) qui rusent avec les procédures de l'appropriation (l'auteur-éditeur ou l'auteur-correcteur), ou qui, pour les mêmes raisons, troublent celles de l'attribution ${ }^{29}$ - le nom d'auteur ${ }^{30}$ étant, par le biais de l'anonymat, du pseudonymat (Witkiewicz), du cryptonymat, de l'hétéronymat, l'un des objets privilégiés de ces jeux de dissimulation.

Il s'agit souvent moins, cependant, pour les figures dont il est ici question, de distendre ou de fausser ces rapports que de les interroger, d'en montrer le caractère problématique, de faire apparaître la complexité des opérations qui les établissent, et que méconnaissent [voir $\mathrm{H}$. Maurel-Indart, infra] les évidences de la doxa. Toutes les relations qui construisent cet " être de raison ${ }^{31}$ " qu'est l'auteur sont, dans les textes ici évoqués, passées au crible de la réflexion fictionnelle: celle de l'auteur et de l'œuvre ("unité première" ${ }^{32}$ ", pour M. Foucault, dans le contexte d'une individualisation des discours) fait l'objet d'un questionnement récurrent, qui souligne l'incertitude ou la fragilité de ce lien (Pinget, Calvino...), ou la difficile distinction des deux termes (Vila-Matas) - ce démontage systématique ne va pas sans conduire à une paradoxale affirmation d'auctorialité (Borges, par exemple). Ce lien primordial se décompose en un faisceau de relations qui donne matière à investigation. L'étymologie fait de l'auteur le garant de son œuvre: sont dès lors interrogés les rapports du scriptor et de l'auctor (Nodier, Nerval, Pinget, Calvino); surgit, de même, la question de l'intention de l'auteur: comme principe herméneutique (Gac), comme principe de détermination - on touche au problème de la nécessité de l'œuvre, de la manière dont elle se soustrait, ou non, au hasard (Mallarmé, Borges, Cortázar, Calvino) ; ou encore - dans une tradition de biographisation de l'écrivain inaugurée par le romantisme - celle du rapport de l'œuvre à la vie de son auteur ${ }^{33}$ (Gac); l'auteur, c'est aussi ce

28. Sur les paradoxes qui entrent en jeu dans la constitution du portrait de l'auteur (contradiction entre être et paraître, dire et montrer, désir d'être publié et refus d'être connu...) voir Portraits de l'écrivain contemporain, textes réunis et présentés par J.-F. Louette et R.-Y. Roche, Champ Vallon, 2003.

29. M. Foucault, art. cit.

30. Ibid.

31. Ibid, p. 801 .

32. Ibid., p. 792.

33. D. Maingueneau appelle "paradoxe d'Achab" la relation d'enveloppement réciproque 
principe de cohérence, qu'affirment, pour mieux le dénoncer, les films de Lynch; ou celui de l'"unité d'écriture ${ }^{34}$ », que l'on voit atteint de soupçon (mais néanmoins revendiqué) dans le thêâtre de Pinget... La relation de l'auteur au lecteur est explorée de même, et montrée tout aussi sujette à ces brouillages de hiérarchies et de frontières qui affectent la relation de l'auteur à l'œuvre et déterminent ses paradoxes: l'usage du pseudonyme, qui se fait surnom (Witkiewicz/Witkacy), est une manière de faire intervenir cet Autre qu'est le lecteur dans la construction de l'identité auctoriale, et de faire perdre à la figure de l'auteur ses contours nets; dans la démarche d'interprétation, ou l'acte de compréhension, le spectateur de cinéma (Lynch), comme le lecteur de roman (Gac) se fait auteur d'une fiction - alors que l'auteur luimême, si on le ressuscite, ne peut se lire (Gac)...

Un autre paradoxe fondamental se trouve révélé par nos figures critiques. Il réside, cette fois, dans la relation de l'écrivain moderne à l'autorité: entendons par là cette légitimité qui se fonde, jusqu'au classicisme, sur la participation à une tradition (l'auctoritas des Anciens est conférée à des auctores, garants, modèles, et références, dans un régime de savoir qui repose sur l'assignation d'une connaissance à un nom propre - "Aristoteles dixit...») et s'accorde à "ceux qui, par leur état, ont pouvoir d'énoncer la vérité35". Le Romantisme, à cet égard, marque un tournant décisif dans l'histoire de l'auctorialité. Le régime de l'autorité fait place désormais à celui de l'authorshi $p^{36}$ : c'est la singularité du créateur qui se trouve à présent valorisée, et l'œuvre vaut par son originalité, par ce qui précisément la distingue de toute autre. L'après-Révolution, il faut le rappeler, a détruit les corporations de l'Ancien Régime, qui donnaient à l'" homme de lettres» son état, et l'autorisaient en sa qualité d'homme d'étude et de savoir, ainsi que les réseaux sociaux dans lesquels il se trouvait intégré en amont de la publication ${ }^{37}$. L'ère nouvelle s'ouvre ainsi sur une crise de légitimité de l'écrivain. Il doit désormais tirer cette légitimité de lui-même, et son discours d'individu "n'a d'autre valeur

qui s'instaure entre l'œuvre et la vie d'un écrivain (Le Contexte de l'auvre littéraire, op. cit., p. 6I).

34. M. Foucault, art. cit, p. 802.

35. R. Chartier, op. cit., p. 72 (nous soulignons).

36. La distinction est faite par G. Leclerc, Le Sceau de l'auvre, Seuil, 1998, p. 103 sq.

37. Voir A. Vaillant, "Entre personne et personnage. Le dilemme de l'auteur moderne", L'Auteur. Colloque de Cerisy-la-salle, actes publiés sous la direction de G. Chamarat et A. Goulet, Presses universitaires de Caen, 1996. La légitimation nouvelle viendra de l'institution de la littérature, qui se constitue au cours du premier dix-neuvième siècle. Celle-ci ne teconduit évidemment pas le régime de l'auctoritas, même si l'autorité ne disparaît pas totalement des textes littéraires modernes (voir A. Brunn, op. cit., p. 36). 
que celle de l'individualité qui le soutient et l'autorise ${ }^{38}$ ". Les figures ici rassemblées signifient, de fait, une formidable déflation de l'autorité; celle-ci est à chercher maintenant dans des champs du savoir extérieurs à la littérature (Balzac). Elles sont, pour la plupart, délégitimantes, et ne manquent pas (on retrouve ici la valeur critique du paradigme du faux) d'interroger cette dimension essentielle de l'auctoritas: la faculté de tenir un discours de vérité (Nerval, Pinget, Calvino, Lynch). Il semble ainsi que l'auctorialité moderne se construise sur une négation de l'autorité - que, pourtant, elle conserve comme référence.

Dans bien des cas, c'est par la négation de cette négation que l'écrivain trouve à s'autoriser (dans le jeu de renversement, déjà noté, par lequel il se défait de cette sorte de négativité qui l'affecte $a$ priori)... Dans sa quête de légitimité, il se désigne volontiers comme fils de son œuvre (Schwob, Gac), inversant le rapport de filiation attendu, ainsi que le processus d'indexation qui est au principe même de l'auctoritas. Cette tendance de l'auctorialité à se décider, dans le rapport de filiation, en aval, se retrouve à une échelle plus large, dans un phénomène qui apparaît comme spécifique aux deux siècles qui suivent la fracture révolutionnaire - et par rapport auquel on peut penser, en saisissant son émergence dans une durée longue, la théorie de la " mort de l'auteur": la mise en place d'une "autorité à rebours" [A. Brunn, infra]. La source de l'autorité se trouve à présent chez le lecteur, qui a pouvoir d'en créditer l'auteur; celle-ci remonte du commentateur au texte commenté, au lieu d'en émaner comme c'était le cas dans le précédent régime d'auctorialité (Cousin). Le lecteur profite, comme on vient de l'entrevoir (à sa manière, $\mathrm{R}$ Barthes ${ }^{39}$ avait rendu compte de ce déplacement), d'un appel d'auctorialité créé par un évidement de la fonction auctoriale. La cause s'en trouve dans le brouillage ou la dissolution de l'origine, phénomène historique qui marque l'entrée dans l'ère post-révolutionnaire, et par rapport auquel prend pleinement sens le questionnement évoqué plus haut. Mais c'est sous les espèces du critique, précisément, que le lecteur se trouve ainsi promu: l'on voit, dans cette mutation, surgir une autorité concurrente, une autre figure paradoxale de l'auteur, et des plus prégnantes, en raison des enjeux, majeurs, qui se cristallisent désormais autour d'elle (Cousin, Pinget, Cortázar...).

C'est comme une figure ironisée qu'apparait ainsi, dans notre corpus de textes, la figure de l'auteur: spectre d'une autorité devenue problématique (mais qui ne cesse, sous ces formes altérées ou inversées, de faire retour), elle

38. M. Condé, La Genèse sociale de l'individualisme romantique, Tübingen, Max Niemeyer Verlag, 1989, p. II.

39. Art. cit., p. 45 
doit se constituer à partir de tant d'exigences, parfois contradictoires, qu'ellc semble ne pouvoir émerger sous une forme immédiatement et pleinement positive (ou conforme aux représentations de la doxa). Étendant le questionnement, on pourrait se demander s'il existe des figures ${ }^{40}$ de l'auteur qui échappent totalement à la logique du paradoxe...

Elle n'a cessé de nous apparaître de même comme une figure ironisante, qui, dans la mesure même où elle exhibe les antinomies sur lesquelles elle se construit, et souligne les apories de sa propre représentation, interroge, de l'intérieur des textes, la sérieuse fonction-auteur. Brouillant à plaisir des frontières que l'on pourrait croire bien tracées, elle révèle l'instabilité d'une notion que l'imaginaire collectif rend monolithique et naturalise volontiers. Parce qu'elle n'a précisément aucune naturalité, l'auctorialité doit s'affirmer de manière oblique, par le biais, notamment, de ces jeux spéculaires qui se donnent ici libre cours.

Ces quelques remarques générales ne prétendent pas rendre compte de la diversité des jeux de la figure auctoriale avec le paradoxe. Ils n'épargnent ni les fondements juridiques de l'auctorialité, ni ses aspects institutionnels (professionnalisation de l'écriture, autonomisation du champ, conquête des places de discours). Ils sont à observer dans les systèmes qu'ils proposent, dès lors que sont réunies, dans une même œuvre, comme c'est souvent le cas (Calvino, Goscinny, Lynch...), plusieurs figures d'auteur. Ils sont à suivre dans leurs modifications et leurs surenchères, sous l'influence des contextes divers qui les font se déployer, tout au long de la période considérée. Du début du XIX' siècle à la littérature la plus contemporaine, française ou étrangère, les figures convoquées ne sont pas les mêmes, et, quand elles perdurent, elles occupent différemment le devant de la scène, ou changent de signification. Aussi l'ordre dans lequel sont présentées les différentes contributions s'efforce-t-il de respecter, dans l'ensemble, la chronologie et d'en accentuer les grandes articulations. La période examinée a son unité, mais elle est aussi marquée, au tournant des $\mathrm{XIX}^{c}$ et $\mathrm{XX}^{c}$ siècles, par une rupture: pour l'histoire littéraire, un nouveau régime d'auctorialité s'ouvre, on le sait bien, avec Mallarmé; on verra comment il est annoncé par des transformations qui se produisent dès le début des années 1850; on assistera sans surprise, par ailleurs, aux manifestations du "retour de l'auteur" qui caractérisent (bien que celui-ci, évidemment, n'ait jamais disparu!) la fin du $\mathrm{XX}^{\mathrm{c}}$ siècle. Une réflexion sur des problèmes de littérature légale viendra clore la série des lectures contemporaines, avant que deux articles n'élargissent la perspective, en

40. Textuelles, encore une fois. 
abordant des problématiques spécifiques à deux autres arts: la bande dessinée et le cinéma. La B.D. "d'auteur» vient à l'existence et se légitime en déconstruisant les postures auctoriales de la "grande " littérature. Au cinéma, la figure de l'auteur est essentiellement paradoxale : elle est une absence radicale en même temps qu'un point de vue omniprésent, et c'est de ce paradoxe même qu'elle peut tirer son pouvoir...

Ce volume, issu d'une réflexion menée par l'équipe É.CRI.RE. (Équipe de recherche sur la crise de la représentation) au cours des années 2000 à 2002, intègre, notamment, les actes d'une journée d'étude qui s'est tenue à l'université Stendhal ${ }^{4 x}$ et en a été un temps fort. Il fait suite à trois autres publications de cette équipe, retraçant les étapes d'une recherche sur les rapports entre argumentation et fiction ${ }^{42}$. Certains articles de ce numéro poursuivent une investigation entreprise dans le troisième volet de ce programme précédent ("Critique et fiction").

41. Le 13 juin 2002.

42. "Que prouve la littérature? Fiction et argumentation *, textes réunis et présentés par L. Queffélec-Dumasy et J.-F. Louette, Dix-neuflvingt, Revue de littérature moderne, $\mathrm{n}^{\circ} 3$, mars 1997; "De l'argumentation à la fiction. Passages", textes recueillis et présentés par L. Dumasy, Grenoble 3, Recherches \& Travaux $\mathrm{n}^{\circ}$ 57, 2000; "Critique et fiction", textes recueillis et présentés par A.-M. Monluçon, Grenoble 3, Recherches \& Travaux n 60, 2002. 